\title{
Approximation of Bidirectional Reflectance Distribution Function with 3-Degree Polynomial
}

\author{
A. Romanyuk \\ "Vinnitsia National Technical University", \\ Faculty of Computer Intelligence \\ 95, Hmelnitske Shosse street, Vinnytsia, 21021, Ukraine \\ ran@svitonline.com
}

\begin{abstract}
The purpose of this article is approximation of BRDF with functions of argument $\cos (x)$. The described approach proposes usage of two functions for BRDF approximation. One of them is a cubic polynomial (with argument $\cos (x))$ and the second one is the function, that assures monotonous falling of the function. Usage of this approach leads to considerable reduce of the approximation error and increases accuracy of the object's highlights representation.
\end{abstract}

Index Terms - Shading, BRDF, Bidirectional Reflectance Distribution Function, illumination model, BRDF approximation.

\section{INTRODUCTION}

The most complicated and resource-intensive computing at the rendering stage takes place in the shading of 3-D objects, which have voluminosity effect created by color gradation. The color intensity and coordinates are detected for every pixel on the surface in the process of shading. Taking into consideration, that high resolution displays are used for representing realistic images, shading process takes a lot of time. Especially it reveals when illumination models are used, in particular that which represent specular constituent of the color. That's why the question of the increasing shading production in computer graphic systems is very urgent one.

\section{Existing Approaches ANALysis AND Problem Formulation}

The intensity of pixels color according to Phong method is de-

$$
1-4244-0346-4 / 07 / \$ 20.00 \text { (C) } 2007 \text { IEEE }
$$


tected with using following formula:

$$
I=\mathrm{I}_{\mathrm{a}} \mathrm{k}_{\mathrm{a}}+\mathrm{I}_{1}\left(\mathrm{k}_{\mathrm{d}} \cos \psi+\mathrm{k}_{\mathrm{s}} \cos ^{\mathrm{n}} \lambda\right),
$$

where $I_{a}, I_{l}$ are intensities of sparse and directed light sources correspondingly, $k_{a}, k_{d}, k_{s}$ is sparse, diffusive and reflecting light coefficients, $\psi$ is angle between the direction of light and normal vector, $\lambda$ is angle between the direction of reflected light and the observer, $n$ is surface brightness coefficient, $\cos ^{n} \lambda$ is BRDF, represents surface optical properties.

In shading of the surfaces, the most resource-intensive procedure is a computing $\cos ^{n} \gamma$, that is used in Phong and Blinn illumination models [1]. It is to be mentioned, that $n$ varies in diapason from 0 to 1 . K. Schlick [2] proposed approximation of the function $\cos ^{n} \gamma$, which is used for computing intensity of the mirror constituent of the color in Phong and Blinn illumination models, with function

$$
\cos \gamma /(n-n \cos \gamma+\cos \gamma)
$$

Analysis showed this approach to provide satisfactory quality only of highlight's epicenter representation. Beyond this area discrepancy with results, received according to Blinn illumination model is observed.

Function $\cos ^{n} \gamma$ is expanded into Taylor series in method [3], proposed by R.F.Lion. Instead of the angle $\lambda$ between reflected light and observer, the length of chord between mentioned vectors is used:

$$
\gamma=|\vec{D}|=|\vec{N}-\vec{H}| .
$$

Expression $1 / \sqrt{\vec{N} \cdot \vec{N}}$, which is used for normalizing of normal vector $\vec{N}$, is also expanded into Taylor series. Only first 3 terms are used. This allows exclusion of division and square root operations from computing process. Replacement of the angle with chord length doesn't influence greatly upon accuracy of the calculations only for low values of angles, and using limited quantity of Taylor series 
doesn't allow to perform normal vectors normalizing and $\cos ^{n} \lambda$ approximation with necessary accuracy.

Rather better accuracy of approximation is reached in approximation of function $\cos ^{n} \lambda$ with function [4] $\cos ^{k}(\sqrt{n / k} \cdot \lambda)$, where $k=2,4,8, \ldots$ and is selected depending on diapason of $n$. Unfortunately, for this approach it is necessary to define angle $\lambda$, which envisages performing resource-intensive operation of arcos.

The purpose of the article - BRDF approximation with 3-degree polynomial with condition, that input operand is a cosine between normal vector and $\vec{H}$ vector.

\section{MAIN STATEMENT}

Let's approximate BRDF with cubic following polynomial $f(x)$ :

$$
f(x)=a \cdot \cos (x)^{3}+b \cdot \cos (x)^{2}+c \cdot \cos (x)+d=\cos (x)^{n}
$$

For finding $a, b, c, d$ it is considered, that $\cos (0)=1$, so that :

$$
a+b+c+d=1
$$

If $\cos (x)=0$ then

$$
d=0
$$

Let's equate value of BRDF and value of cubic polynomial at the point $t$, where $\cos (t)^{n}=0.5$. Taking into consideration, that $\cos (t)=\sqrt[n]{0.5}$, we receive:

$$
a \cdot \cos (t)^{3}+b \cdot \cos (t)^{2}+c \cdot \cos (t)+d=0.5
$$

The last equation for finding variables $a, b, c, d$ can be received by equating derivatives of BRDF and proposed cubic polynomial at the point $t$, where $\cos (t)^{n}=0.5$.

$$
\begin{gathered}
B R D F^{\prime}=-n \cdot \cos (x)^{n-1} \cdot \sin (x) \\
f(x)^{\prime}=-3 \cdot a \cdot \cos (x)^{2} \cdot \sin (x)--2 \cdot b \cdot \cos (x) \cdot \sin (x)-c \cdot \sin (x)
\end{gathered}
$$

Equating right parts of terms (5) i (6) and dividing to 
$-\sin (x)$ gives:

$$
n \cdot \cos (t)^{n-1}=3 \cdot a \cdot \cos (t)^{2}+2 \cdot b \cdot \cos (t)+c
$$

The equations $2,3,4,7$ compose system of equations for defining coefficients of cubic polynomial (1):

$$
\left\{\begin{array}{l}
a+b+c+d=1 \\
d=0 \\
a \cdot \cos (t)^{3}+b \cdot \cos (t)^{2}+c \cdot \cos (t)+d=0.5 \\
n \cdot \cos (t)^{n-1}=3 \cdot a \cdot \cos (t)^{2}+2 \cdot b \cdot \cos (t)+c
\end{array}\right.
$$

After equivalent transformation the system of two equations is received:

$$
\left\{\begin{array}{l}
a \cdot \cos (t)^{3}+b \cdot \cos (t)^{2}+(1-a-b) \cdot \cos (t)=0.5 \\
n \cdot \cos (t)^{n-1}=3 \cdot a \cdot \cos (t)^{2}+2 \cdot b \cdot \cos (t)+(1-a-b),
\end{array}\right.
$$

where $\cos (t)=\sqrt[n]{0.5}$.

Solution of the last system of equations is expressed in the following formulas:

$$
\begin{aligned}
& a=0.5 \cdot \frac{-2 q+1+2 q^{2}+n \cdot q-n}{q^{2}\left[-2 q+q^{2}+1\right]}, \\
& b=-0.5 \frac{4 q^{3}+q^{2} \cdot n-3 q^{2}-n+1}{(q-1)^{2} \cdot q^{2}},
\end{aligned}
$$

where $q=e^{\frac{-0.6931471}{n}}$.

It is advisable to store coefficients $a$ and $b$ for all possible values of $n$ in permanent memory unit.

An example of BRDF approximation with cubic polynomial is represented in the figure 1 . It is shown at the graphic, that using cubic polynomial for highlights fading area is impossible, "cause increasing of the specular constituent of light is observed instead of fading.

Modeling has shown that for $\forall n \in[1 ; 256], \Rightarrow \delta(n) \leq 0.67 \%$ (fig. 2).

In comparison with Schlick model of BRDF at this interval relative error is decreased more than in 17 times. It is worth to be men- 
tioned, that in BRDF approximation the most exacting requirements are set for representation of highlight's epicenter [2], which occurs above cusp. For other areas it is enough to provide monotonous fading of color intensity, which excludes appearance of artifacts [2, 5]. The requirements to accuracy are not exacting as a rule [6].

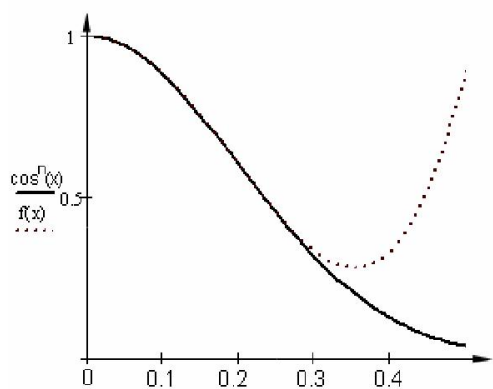

Fig. 1. An Example of BRDF approximation for $n=25$

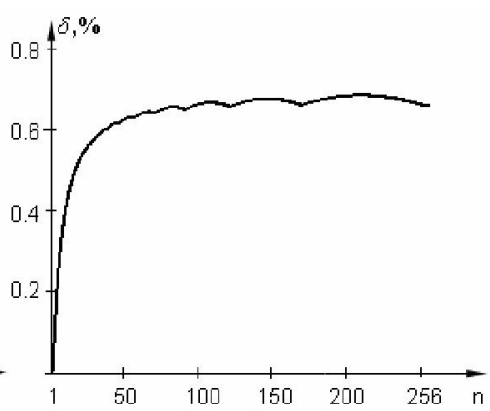

Fig. 2. Graphic of maximal relative error of BRDF approximation

Usage of BRDF approximation with cubic polynomial provided that $\cos ^{n}(x)<0.5$ can lead to such an undesirable result, represented on figure 3. It's kernel is that BRDF takes on negative values even if contingencies are provided that $\cos ^{n}(x)>0.5$.

Let's analyze BRDF like $g(x)=\frac{\cos (x)}{a-b \cos (x)}$ if $\cos ^{n}(x)<0.5$ is provided. The coefficients $a, b$ can be found by equating values of $g(x)$ and $\cos ^{n}(x)$ at the point $x=\arccos (\sqrt[n]{0.5})$, and of their derivatives at this point. As a result following system of equations is received:

$$
\left\{\begin{array}{l}
\frac{\cos (t)}{a-b \cdot \cos (t)}=0.5 \\
\frac{1}{a-b \cdot \cos (t)}+\frac{\cos (t) \cdot b}{\left(a-b \cdot \cos ^{2}(t)\right)}=n \cdot \cos ^{n-1}(t)
\end{array}\right.
$$


where $\cos ^{n}(t)=0.5$

Let's show, that $b=2 \cdot n-2, a=2 n \sqrt[n]{0.5}$. The solution of the system of equations is:

$$
\left(\begin{array}{l}
a \\
b
\end{array}\right)=\left(\begin{array}{l}
4 \cdot n \cdot \cos ^{n+1}(t) \\
4 \cdot n \cdot \cos ^{n}(t)-2
\end{array}\right) .
$$

In view of $\cos ^{n}(t)=0.5$, wherefrom $\cos (t)=\sqrt[n]{0.5}$ it is resulted in:

$$
\left(\begin{array}{l}
a \\
b
\end{array}\right)=\left(\begin{array}{l}
2 \cdot n \cdot \sqrt[n]{0.5} \\
2 \cdot n-2
\end{array}\right)
$$

The $g g(x)$ function is represented on figure 4 , - example of BRDF approximation with couple of functions.

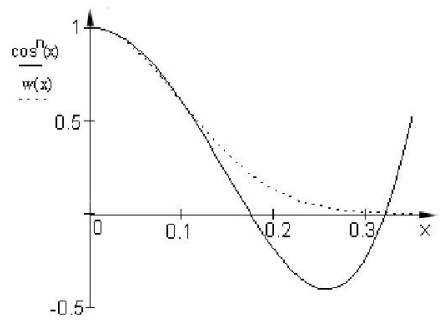

Fig. 3. The weakness of using cubic polynomial

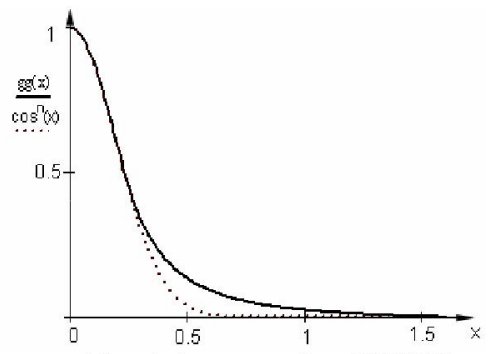

Fig. 4. An example of BRDF approximation with functions $f(x), g(x)$ for $n=25$

The $g g(x)$ function is represented on figure 4, - example of BRDF approximation with couple of functions. It is to be mentioned, that relative error of function $g(x)$ representing highlights fading area, doesn't exceed $1 \%$ if $0.42<f(x)<0.5$, doesn't exceed $3 \%$ if $0.37<f(x)<0.42$ and is less than $10 \%$ if $0.27<f(x)<0.37$, that is acceptable. 


\section{CONCLUSIONS}

New BRDF approximation, being proposed in this work, represents highlights on the surface of graphic objects with rather high accuracy, at the same time approximating function operates with cosine of angle between vector of normal $\mathrm{N}$ and middle vector $\mathrm{H}$. In comparison with Schlick BRDF considerable reduction of approximation error is reached both in highlight epicenter and fading area representation.

\section{REFERENCES}

[1] D. Hern, pavlin Baker M., "Computer Ghaphics and Standard OpenGL. Moscow, 2005. 1168 p.

[2] Chrictophe Schlick, "A Fast Alternative to Phong's Specular Model". Graphics Gems IV. Academic Press. 1994, pp. 404-409.

[3] R.F. Lyon, "Phong Shading Reformulation for Hardware Renderer Simplification". Apple Technical Report №43, 1993.

[4] Романюк О.Н, Чорний А.В. Новий підхід до визначення спекулярної складової кольору // Оптико - електронні інформаційно енергетичні технології. - 2004. pp. 85-92.

[5] C.F. Chang, Bishop G., Lastra A. LDI Tree: A Hierarchical Representation for Image-Based Rendering // In Computer Graphics, SIGGRAPH '99 Proceedings. - 1999. - pp. 291-298

[6] Foley, Van Dam, Feiner, and Hughes, "Computer Graphics". Principles and Practice. Addison Wesley. Ch. 16. 1996, pp. 800-870. 